\title{
A meta-analysis of case studies and clinical characteristics of hypertrophic olivary degeneration secondary to brainstem infarction
}

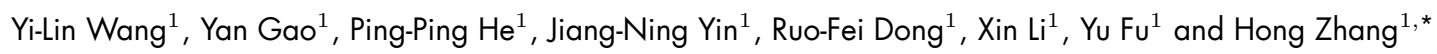

\author{
${ }^{1}$ Department of Neurology, Shengjing Hospital of China Medical University, No. 36 Sanhao Street, Heping District, \\ Shenyang, Liaoning, 110004 , P. R. China \\ *Correspondence: hongzhang@cmu.edu.cn (Hong Zhang)
}

DOI: $10.31083 /$ i.jin.2020.03.1238

This is an open access article under the CC BY-NC 4.0 license (https://creativecommons.org/licenses/by-nc/4.0/).

Transsynaptic degeneration in the cerebellum and brainstem may give rise to a rare neurological condition with various clinical manifestations, namely hypertrophic olivary degeneration. The classical manifestations of hypertrophic olivary degeneration comprise myoclonus, palatal tremor, ataxia, and ocular symptoms. Any lesions interrupting the dentate-rubro-olivary pathway, referred to as the anatomic Guillain-Mollaret triangle, contribute to the broad aetiologies of hypertrophic olivary degeneration. The clinical diagnosis depends primarily on the associated symptoms and the characteristic magnetic resonance imaging findings. Concerning treatment and prognosis, there are no widely accepted guidelines. Here, we identified 11 cases of hypertrophic olivary degeneration secondary to brainstem infarction from 1964 to the present. Combined with two of our cases, the clinical and imaging findings of 13 patients with hypertrophic olivary degeneration secondary to brainstem infarction were studied. A meta-analysis of case studies gives the correlation coefficient between infraction location and time to develop hypertrophic olivary degeneration as $0.217(P=0.393$, $P>0.05)$. At the significance level of $P<0.05$, there was no significant correlation between infraction location and time to develop hypertrophic olivary degeneration. The $\chi^{2}$ between infraction location and magnetic resonance imaging findings of hypertrophic olivary degeneration was $8.750(P=0.364, P>0.05)$. At the significance level of $P<0.05$, there was no significant correlation between infraction location and magnetic resonance imaging findings of hypertrophic olivary degeneration. Conclusion based on the analysis of available data suggests that when newly developed or progressive worsening motor symptoms are presented in patients with previous brainstem infarction, a diagnosis of hypertrophic olivary degeneration should be investigated.

\section{Keywords}

Hypertrophic olivary degeneration; brainstem; Guillain-Mollaret triangle; inferior olive nucleus; magnetic resonance imaging; neurological dysfunction

\section{Introduction}

Hypertrophic olivary degeneration (HOD) is a rare neuronal degenerative condition involving the inferior olivary nucleus (ION) (Sen et al., 2014). It usually results from a lesion along the dentate-rubro-olivary pathway, referred to as the GuillainMollaret triangle (GMT) (Yun et al., 2013). The GMT consists of the dentate nucleus in the cerebellum, the contralateral ION in the medulla oblongata, and the contralateral red nucleus at the level of the midbrain (Menendez et al., 2014). HOD is an unusual entity, as degeneration is accompanied by hypertrophy rather than the more commonly encountered atrophy (Sen et al., 2014). Histologically, the characteristics of HOD are glial demyelination, proliferation, neuronal vacuolation, and shrinkage (Meoded et al., 2013).

Hemorrhagic lesions (Wein et al., 2015), ischemic lesions (Sabat et al., 2016), and neurosurgical intervention of a brain tumor (Shinohara et al., 2013) involving the GMT are common causes of HOD. Moreover, HOD has been reported in head trauma, subarachnoid hemorrhage (Hayashi et al., 2014), and a few mitochondrial disorders (Bindu et al., 2014) such as the Leigh syndrome (Sonam et al., 2014). Studies have reported that an intact dentaterubro-olivary pathway has been detected in a minority of patients with HOD (Van Eetvelde et al., 2016). Additionally, HOD can also be an occasional imaging finding in some asymptomatic patients, for which the underlying mechanism remains unclear and requires further research (Van Eetvelde et al., 2016). We present magnetic resonance imaging and clinical findings for two patients with HOD secondary to brainstem infarction, as well as a meta-analysis of 11 cases of HOD secondary to brainstem infarction from 1964 to the present in the literature.

\section{Case reports}

\subsection{Case report 1}

A 67-year-old woman presented with ataxia. 5 months before hospital admission, she had experienced a loss of consciousness, as well as urinary and fecal incontinence, and was diagnosed with acute infarction of the medulla oblongata. After a week of treatment, she had residual gait unsteadiness. Neurological examination showed a positive Romberg's sign and abnormal heel-to-shin and finger-to-nose results. Additionally, there was bilateral vertical nystagmus with an upward gaze. The remaining neurological 
symptoms were unremarkable. On her postinfarction cranial magnetic resonance imaging (MRI) examination at 5 months, slight enlargement and prominent $\mathrm{T} 2$ hyperintensity were observed for both IONs (Fig. 1). A diagnosis of HOD was made based on clinical manifestations and the results of imaging examinations. She received symptomatic therapy, which included aspirin, statin, and neuroprotective drugs, for about one week without any apparent substantial symptom alleviation. At the 4-year follow-up, she reported aggravation of the unsteady gait. She complained that she could not stand by herself because of poor balance.

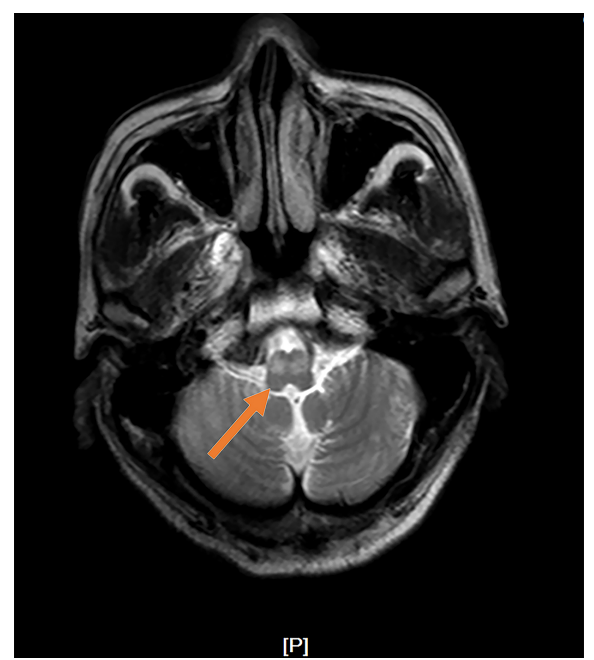

Fig. 1. Increased signal intensity and enlargement of the bilateral inferior olivary nuclei (ION) (yellow arrow) resulting from brainstem infarction.

\subsection{Case report 2}

A 61-year-old man presented with ataxia, slurred speech, diplopia, and choking without any triggers, had a previous episode of these symptoms 7 months before hospital admission when he was diagnosed with an acute infarction of the pons and had residual unstable gait. Neurological examination revealed slurred speech, diplopia, and abnormal finger-to-nose, heel-to-shin, and Romberg's sign test results. The remaining neurological signs were unremarkable. In a follow-up, MRI examination performed 7 months after the infarction, enlarged, and remarkable T2 hyperintense bilateral olives were observed (Fig. 2). Combined with the classical MRI findings and symptoms, a diagnosis of HOD was made. He received aspirin, statin, and neuroprotective drugs for about 8 days, without apparent substantial symptom alleviation. The patient reported no obvious symptom alterations and no appearance of new manifestations at the 1-year follow-up.

\section{A meta-analysis of case studies}

We conducted a meta-analysis of case studies of HOD after brainstem infarction and included cases reported from 1964 to the present. The meta-analysis of case studies were performed utilizing NCBI-PubMed using as keywords "hypertrophic olivary degeneration." We also reviewed the bibliography of the selected articles. As shown in Table 1, of the 13 reported cases of brainstem infarction followed by HOD, 7 (53.8\%) were male, 6 (46.2\%)

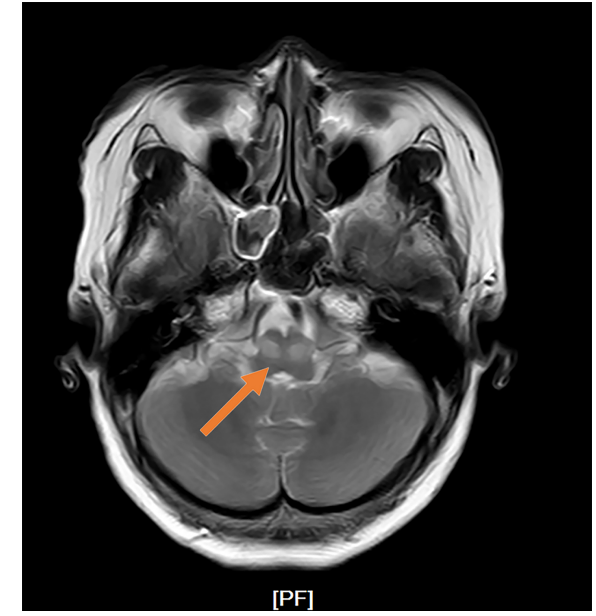

Fig. 2. Bilateral hypertrophic olivary degeneration and increased signal intensity (yellow arrow) secondary to brainstem infarction on the T2-weighted sequence on magnetic resonance imaging (MRI).

were female, the pons was involved in 9 cases $(69.2 \%)$, bilateral olivary nuclei were implicated in 8 cases $(61.5 \%)$, 4 cases $(30.8 \%)$ were unilateral, 1 case was not reported. Ataxia (7 cases, 53.8\%) was the most frequently observed clinical manifestation, followed by palatal myoclonus (5 cases, $38.5 \%$ ) and ophthalmoplegia (5 cases, $38.5 \%$ ), the next was nystagmus ( 4 cases, $30.8 \%$ ). The time to develop HOD varied from several months to several years, the median of which was 4.5 months. There was little literature mentioned treatment and prognosis, and currently, there were no specific drugs for HOD with poor prognosis.

As shown in Table 2, regarding infraction location and time to develop HOD, we performed Spearman's correlation analysis, and the results show that the correlation coefficient between infraction location and time to develop HOD was $-0.217(P=0.393$, $P>0.05)$. At the significance level of $P<0.05$, there was no significant correlation found between infraction location and time to develop HOD. Regarding the infraction location and MRI findings of HOD, we performed Chi-Square analysis. As shown in Table 3 and Table $4, \chi^{2}=8.750(P=0.364, P>0.05)$. At the significance level of $P<0.05$, there was no significant correlation between infraction location and MRI findings of HOD.

\section{Clinical characteristics \\ 4.1 Gender predominance and location}

As shown in Table 1, of the 13 reported cases, 7 (53.8\%) were male, 6 (46.2\%) were female, no apparent gender differences were observed. Bilateral olivary nuclei were implicated in 8 cases $(61.5 \%)$, and 4 cases (30.8\%) were unilateral, which is in line with the available literature that a bilateral process was the most common presentation of the HOD (Carr et al., 2015). In Table 1, there were 8 patients with bilateral HOD, of whom 5 were female, and 3 were male. While according to literature, bilateral HOD was more frequently observed in males (Konno et al., 2016).

\subsection{Symptoms}

HOD patients usually present with Holmes' tremor, palatal myoclonus, and ocular myoclonus (Sen et al., 2014). Holmes' tremor is defined as a slow $(2-5 \mathrm{~Hz})$ rest and intentional tremor (and pos- 
Table 1. Case series of Hypertrophic Olivary Degeneration Secondary to Brainstem Infarction.

\begin{tabular}{|c|c|c|c|c|c|c|c|c|}
\hline Case & Age & Sex & Etiology/location & Time to Develop HOD (month) & MRI findings & Symptoms & Treatment & Prognosis \\
\hline Case 1 & 60 & $\mathrm{~F}$ & Infarction/Pons & 12 & $\mathrm{~B}$ & Tremor; Hemiparesis & $\begin{array}{l}\text { Symptomatic } \\
\text { therapeutic }\end{array}$ & Poor \\
\hline Case 2 & 43 & $\mathrm{~F}$ & Infarction/Pons & 4 & $\mathrm{~L}$ & Palatal Myoclonus & - & - \\
\hline Case 3 & 69 & $\mathrm{~F}$ & Infarction/Midbrain & 4 & $\mathrm{~B}$ & $\begin{array}{l}\text { Ataxia; Ophthalmo- } \\
\text { plegia; Head tremor; } \\
\text { Holmes' tremor }\end{array}$ & Levodopa & Poor \\
\hline Case 4 & 40 & $\mathrm{M}$ & $\begin{array}{l}\text { Infarction/Pons } \\
\text { and Midbrain }\end{array}$ & 4 & - & $\begin{array}{l}\text { Palatal Myoclonus; } \\
\text { Quadriplegia }\end{array}$ & - & - \\
\hline Case 5 & 60 & M & Infarction/Midbrain & 3 & $\mathrm{~B}$ & $\begin{array}{l}\text { Palatal Myoclonus; } \\
\text { Head tremor }\end{array}$ & - & - \\
\hline Case 6 & 59 & $\mathrm{M}$ & $\begin{array}{l}\text { Infarction/Left } \\
\text { red nucleus }\end{array}$ & 84 & $\mathrm{~L}$ & $\begin{array}{l}\text { Ataxia; Ophthalmo- } \\
\text { plegia; Nystagmus }\end{array}$ & - & - \\
\hline Case 8 & 50 & M & $\begin{array}{l}\text { Infarction/Pons } \\
\text { and Midbrain }\end{array}$ & 3 & $\mathrm{~B}$ & Ataxia & - & - \\
\hline Case 9 & 59 & M & Infarction/Pons & 9 & $\mathrm{R}$ & $\begin{array}{l}\text { Ataxia; Palatal My- } \\
\text { oclonus; Nystagmus; } \\
\text { Hemiparesis }\end{array}$ & - & - \\
\hline Case 10 & 50 & M & Infarction/Pons & - & $\mathrm{R}$ & Ataxia; Tremor & - & - \\
\hline Case 11 & 39 & $\mathrm{~F}$ & $\begin{array}{l}\text { Infarction/Pons } \\
\text { and Midbrain }\end{array}$ & 1 & B & $\begin{array}{l}\text { Ophthalmoplegia; } \\
\text { Nystagmus }\end{array}$ & - & - \\
\hline $\begin{array}{l}\text { Current } \\
\text { study }\end{array}$ & 67 & $\mathrm{~F}$ & $\begin{array}{l}\text { Infarction/Medulla } \\
\text { Oblongata }\end{array}$ & 5 & B & $\begin{array}{l}\text { Ataxia; Ophthalmo- } \\
\text { plegia; Nystagmus }\end{array}$ & $\begin{array}{l}\text { Aspirin; } \\
\text { Statin }\end{array}$ & Poor \\
\hline $\begin{array}{l}\text { Current } \\
\text { study }\end{array}$ & 61 & M & Infarction/Pons & 7 & $\mathrm{~B}$ & $\begin{array}{l}\text { Ataxia; Ophthalmo- } \\
\text { plegia }\end{array}$ & $\begin{array}{l}\text { Aspirin; } \\
\text { Statin; Neu- } \\
\text { roprotective } \\
\text { drugs }\end{array}$ & Poor \\
\hline
\end{tabular}

F: Female; M: Male; HOD: hypertrophic olivary degeneration; B: bilateral; L: left; R: right; Case1 (Sen et al., 2014); Case2 (Van Eetvelde et al., 2016); Case 3 (Cosentino et al., 2016); Case 4 (Venkatesh et al., 2017); Case 5 (Zhou et al., 2017); Case 6 (Smets et al., 2017); Case 7 (Santos et al., 2015); Case 8 (Lai et al., 2018); Case 9 (Bruno and Wooten, 2012); Case 10 (Choh et al., 2009); Case 11 (Gerace et al., 2006).

sible postural tremor) (Sen et al., 2014). The rhythmic involuntary movements of the oropharynx are the characteristic feature of palatal myoclonus, which is frequently attributed to the contractions of the levatorveli palatini muscle, and the facial, tongue and larynx muscles are occasionally involved (Van Eetvelde et al., 2016; Wein et al., 2015). Palatal myoclonus is the common manifestation of HOD, while myoclonus is not fundamental to confirm a diagnosis of HOD (Hirano et al., 2015). Additional clinical presentations of HOD vary from progressive ataxia to tremors of additional muscles such as the neck, facial, trunk, pharyngeal, and muscles of the extremities (Meoded et al., 2013). According to Table 1, ataxia was the most frequently observed clinical manifestation in patients with HOD secondary to brainstem infarction, followed by palatal myoclonus and ophthalmoplegia, and the next was nystagmus.

\subsection{Diagnosis and differential diagnosis}

MRI is an essential tool for diagnosing HOD (Cosentino et al., 2016). Diffusion tensor imaging and MR fiber tractography can detect disruptions in the GMT pathways when changes with conventional MRI are equivocal (Sarawagi and Murugesan, 2015). The absence of diffusion restriction, contrast enhancement, and the spatial confinement of the lesion in the vicinity of the ION were valuable contributors to the accurate radiological diagnosis (Van Eetvelde et al., 2016). Inflammatory processes (tuberculosis, encephalitis or sarcoidosis), amyotrophic lateral sclerosis, neoplasia, Wallerian degeneration, adrenoleukodystrophy, and demyelination should be taken into account in the differential diagnosis of HOD (Van Eetvelde et al., 2016).

\subsection{Treatment and prognosis}

Currently, symptomatic treatment is the commonly accepted treatment method with varying results (Orman et al., 2014). Unfortunately, the symptoms of HOD are refractory, and patients usually show an inadequate response to the symptomatic therapeutic approaches, reporting little substantial symptom alleviation (Van Eetvelde et al., 2016). According to Table 1, in general, the prognosis of patients with HOD is poor.

\section{Discussion}

For our patients, ataxia was the shared and main symptom, and neurological examination revealed a positive Romberg's sign and abnormal finger-to-nose and heel-to-shin results, which is consistent with Table 1 that ataxia was the most frequently observed manifestation. It is worth pointing out that given the high recurrence rate of infarction and the low incidence of HOD, the symptoms 
Table 2. Spearman's correlation between infraction location and Time to Develop HOD.

\begin{tabular}{lllcc}
\hline & & Infraction location & Time to Develop HOD \\
\hline \multirow{3}{*}{ Spearman's correlation } & Infraction location & Correlation Coefficient & 1 & -0.217 \\
& & Significance (2-tailed) & & 0.393 \\
& Number of Valid Cases & 13 & 12 \\
& Time to Develop HOD & Correlation Coefficient & -0.217 & 1 \\
& & Significance (2-tailed) & 0.393 & 12 \\
& Number of Valid Cases & 12 & 12 \\
\hline
\end{tabular}

Table 3. Crosstabulation of Chi-Square analysis between Infraction location and MRI findings of HOD.

\begin{tabular}{lccccc}
\hline & & \multicolumn{2}{c}{ MRI findings of HOD } & Total \\
\hline \multirow{4}{*}{ Infraction location } & Bilateral & Left & Right & \\
& Pons & 3 & 1 & 2 & 6 \\
& Midbrain & 2 & 0 & 0 & 2 \\
& Pons and Midbrain & 2 & 0 & 0 & 2 \\
Total & Left red nucleus & 0 & 1 & 0 & 1 \\
& Medulla Oblongata & 1 & 0 & 0 & 1 \\
& & 8 & 2 & 2 & 12
\end{tabular}

Table 4. Chi-Square Tests of Infraction location and MRI findings of HOD.

\begin{tabular}{lccc}
\hline & value & df & $\begin{array}{c}\text { Asymptotic } \\
\text { Significance (2-sided) }\end{array}$ \\
\hline Pearson Chi-Square & 8.75 & 8 & 0.364 \\
Likelihood Ratio & 8.685 & 8 & 0.37 \\
Linear-by-Linear Association & 0.92 & 1 & 0.337 \\
Number of Valid Cases & 12 & & \\
\hline
\end{tabular}

such as ataxia reported by patients may be easily confused with the recurrence of infarction, especially when the common manifestations of HOD such as Holmes' tremor, palatal myoclonus, and ocular myoclonus are not included in the chief complaint. It is of great importance to pay attention to the inferior olivary nucleus when there are no other lesions found in MRI.

For our patients, the time to develop HOD was 5 and 7 months, respectively. Based on the medical history, laboratory tests, cerebrospinal fluid tests, and MRI findings, we ruled out the possibility of other disorders. Combined with the classical MRI findings and symptoms, a diagnosis of HOD was made. Our patients received aspirin, statin, and neuroprotective drugs for about a week without apparent substantial alleviation. The patient of case 1 reported aggravation of the unsteady gait at the 4-year follow-up. In contrast, the patient of case 2 reported no apparent symptom alterations and no appearance of new manifestations at the 1-year follow-up. It is possible that the progression of symptoms is a long and gradual progress, and 1 year is not enough time for our patients to experience apparent changes in symptoms. Alternatively, any alterations in symptoms may have been too subtle.

\section{Conclusions}

When newly developed or progressive worsening motor symptoms are presented in patients with previous brainstem infarction, a diagnosis of HOD should be considered. The clinical diagnosis depends primarily on the associated symptoms and the characteristic MRI findings. Furthermore, there was no significant correlation between infraction location and time to develop HOD. Moreover, there was no significant correlation between infraction location and MRI findings of HOD. Based on the knowledge that the symptoms of HOD are refractory, and there is no effective targeted therapy to date, we believe that new specific treatments are necessary for a satisfactory prognosis.

\section{Author contributions}

Yi-Lin Wang reported the case and drafted the manuscript; Yan Gao, Ping-Ping He, Jiang-Ning Yin, Ruo-Fei Dong, Xin Li, and $\mathrm{Yu}$ Fu reviewed the relevant literature; Hong Zhang reviewed the literature and revised the manuscript. All authors contributed to editorial changes in the manuscript. All authors read and approved the final manuscript.

Ethics approval and consent to participate

This study was approved by the Ethics Committee of China Medical University. Written informed consent for publication of case details and pictures was obtained from each patient.

\section{Acknowledgments}

Thanks to all the peer reviewers and editors for their opinions and suggestions. The authors acknowledge support from Shenyang Science and Technology Project (Project No. 18014482) and 345 Talent Project of Shengjing Hospital of China Medical University.

\section{Conflict of Interest}

The authors declare no conflict of interest.

Submitted: November 15, 2019

Revised: April 17, 2020

Accepted: April 24, 2020

Published: September 30, 2020 


\section{References}

Bindu, P. S., Taly, A. B., Sonam, K., Govindaraju, C., Arvinda, H. R., Gayathri, N., Bharath, M. M., Ranjith, D., Nagappa, M., Sinha, S. Khan, N. A. and Thangaraj, K. (2014) Bilateral hypertrophic olivary nucleus degeneration on magnetic resonance imaging in children with Leigh and Leigh-like syndrome. The British Journal of Radiology 87, 20130478.

Bruno, M. K. and Wooten, G. F. (2012) Hypertrophic olivary degeneration. Archives of Neurology 69, 274-275.

Carr, C. M., Hunt, C. H., Kaufmann, T. J., Kotsenas, A. L., Krecke, K. N. and Wood, C. P. (2015) Frequency of bilateral hypertrophic olivary degeneration in a large retrospective cohort. Journal of Neuroimaging 25, 289-295.

Choh, N. A., Choh, S. A. and Jehangir, M. (2009) Hypertrophic olivary degeneration: the forgotten triangle of Guillain and Mollaret. Neurology India 57, 507-5099.

Cosentino, C., Velez, M., Nunez, Y., Palomino, H., Quispe, D., Flores, M. and Torres, L. (2016) Bilateral hypertrophic olivary degeneration and holmes tremor without palatal tremor: An unusual association. Tremor and Other Hyperkinetic Movements 6, 400.

Gerace, C., Fele, M. R., Luna, R. and Piazza, G. (2006) Neurological picture. Bilateral hypertrophic olivary degeneration. Journal of Neurology, Neurosurgery, and Psychiatry 77, 73.

Hayashi, T., Kigawa, H. and Tanahashi, N. (2014) Hypertrophic olivary degeneration after traumatic subarachnoid hemorrhage. Internal Medicine 53, 1453-1454.

Hirano, M., Hatzoglou, V., Karimi, S. and Young, R. J. (2015) Hypertrophic olivary degeneration resulting from posterior fossa masses and their treatments. Clinical Imaging 39, 787-790.

Konno, T., Broderick, D. F., Tacik, P., Caviness, J. N. and Wszolek, Z. K. (2016) Hypertrophic olivary degeneration: A clinico-radiologic study. Parkinsonism \& Related Disorders 28, 36-40.

Lai, Q., Zheng, C., Zhang, R., Liu, X., Li, Y. and Xu, Q. (2018) MRI and tractography in hypertrophic olivary degeneration. Canadian Journal of Neurological Sciences 45, 332-333.

Menendez, D. F., Cury, R. G., Barbosa, E. R., Teixeira, M. J. and Fonoff, E. T. (2014) Hypertrophic olivary degeneration and holmes' tremor secondary to bleeding of cavernous malformation in the midbrain. Tremor and Other Hyperkinetic Movements 4, 264.

Meoded, A., Poretti, A., Ilica, A. T., Perez, R., Jallo, G., Burger, P. C., Huisman. T. A. and Izbudak, I. (2013) Diffusion tensor imaging in a child with hypertrophic olivary degeneration. The Cerebellum 12, 469474.

Orman, G., Bosemani, T., Jallo, G. I., Huisman, T. A. and Poretti, A. (2014) Hypertrophic olivary degeneration in a child following mid- brain tumor resection: longitudinal diffusion tensor imaging studies. Journal of Neurosurgery: Pediatrics 13, 408-413.

Sabat, S., Mannering, N. and Agarwal, A. (2016) Hypertrophic olivary degeneration: Case series and review of literature. Journal of the Neurological Sciences 370, 180-186.

Santos, A. F., Rocha, S., Varanda, S., Pinho, J., Rodrigues, M., Ramalho Fontes, J., Soares-Fernandes, J. and Ferreira, C. (2015) Hypertrophic olivary degeneration and cerebrovascular disease: movement in a triangle. Journal of Stroke \& Cerebrovascular Diseases 24, e59-e60.

Sarawagi, R. and Murugesan, A. (2015) Hypertrophic olivary degeneration - a report of two cases. Journal of Clinical Imaging Science 5, 8 .

Sen, D., Gulati, Y. S., Malik, V., Mohimen, A., Sibi, E. and Reddy, D. C. (2014) MRI and MR tractography in bilateral hypertrophic olivary degeneration. Indian Journal of Radiology and Imaging 24, 401-405.

Shinohara, Y., Kinoshita, T., Kinoshita, F., Kaminou, T., Watanabe, T. and Ogawa, T. (2013) Hypertrophic olivary degeneration after surgical resection of brain tumors. Acta Radiologica 54, 462-466.

Smets, G., Lambert, J., Tijssen, M., Mai, C., Decramer, T., Vandenberghe, W., Van Loon, J. and Demaerel, P. (2017) The dentato-rubro-olivary pathway revisited: New MR imaging observations regarding hypertrophic olivary degeneration. Clinical Anatomy 30, 543-549.

Sonam, K., Khan, N. A., Bindu, P. S., Taly, A. B., Gayathri, N., M. Bharath, M., Govindaraju, C., Arvinda, H. R., Nagappa, M., Sinha, S. and Thangaraj, K. (2014) Clinical and magnetic resonance imaging findings in patients with Leigh syndrome and SURF1 mutations. Brain \& Development 36, 807-812.

Van Eetvelde, R., Lemmerling, M., Backaert, T., Favoreel, N., Geerts, B., Sommeling, C., Hemelsoet, D. and Dekeyzer, S. (2016) Imaging features of hypertrophic olivary degeneration. Journal of the Belgian Society of Radiology 100, 71.

Venkatesh, M., Prasad, V. R. S., Basha, S. U. and Priya, G. H. J. (2017) Teaching neuroImages: Bilateral hypertrophic olivary degeneration following posterior circulation stroke. Neurology 89, e224-e225.

Wein, S., Yan, B. and Gaillard, F. (2015) Hypertrophic olivary degeneration secondary to pontine haemorrhage. Journal of Clinical Neuroscience 22, 1213-1214.

Yun, J. H., Ahn, J. S., Park, J. C., Kwon, D. H., Kwun, B. D. and Kim, C. J. (2013) Hypertrophic olivary degeneration following surgical resection or gamma knife radiosurgery of brainstem cavernous malformations: an 11-case series and a review of literature. Acta Neurochirurgica 155, 469-476.

Zhou, C., He, Y., Chao, Z., Zhu, Y., Wang, P., Wang, X., Liu, S., Han, W. and Wang, J. (2017) Wernekink commissure syndrome secondary to bilateral caudal paramedian midbrain infarction presenting with a unique "heart or $\mathrm{v}$ " appearance sign: Case report and review of the literature. Frontiers in Neurology 8, 376. 